\title{
Estructura, percepción, factores no-lingüísticos y cuestionamientos de entrevistas OPI en español: Una propuesta de aplicación a la Prueba General de Bachillerato
}

\author{
Luis Guillermo BEJARANO \\ Valdosta State University \\ Nuria OTERO DE JUAN \\ Universidad de Alcalá \\ n.otero@uah.es \\ Jesús GARCÍA LABORDA \\ Universidad de Alcalá \\ jesus.garcialaborda@uah.es
}

Recibido: enero 2012

Aceptado: diciembre 2012

\begin{abstract}
RESUMEN
Este trabajo presenta un estudio sobre la entrevista oral OPI y su aplicación en el diseño de la futura sección de lenguas extranjeras en la Prueba General de Bachillerato (PGB). Tras la presentación contextual de la OPI y la Prueba General de Bachillerato, se presenta un estudio analizando las actitudes de los candidatos de dicho examen y extrayendo las ideas básicas del mismo para aplicarlas al examen español. En la siguiente parte se presenta la integración de lo experimentado en la OPI en la investigación de la PGB, aspecto que queda muy claramente reflejado en las conclusiones donde se realza la experiencia de la OPI para el estudio de las actitudes de los estudiantes españoles.
\end{abstract}

Palabras clave: Español Lengua Extranjera, Lengua extranjera, evaluación, Prueba General de Bachillerato.

Structure, perception, facteurs non linguistiques et questions d'entrevues OPI en Espagnol: Une application proposée au Test General

du Baccalauréat

\section{RÉSUMÉ}

Cet article présente une étude sur le BPR entrevue orale et son application dans la conception de section de langue étrangère dans l'avenir Test General Baccalauréat (PGB). Suite à la présentation du contexte de l'introduction en bourse et de l'École General Test haute, a présenté une étude analysant les attitudes des candidats de cet examen et en extraire les idées de base de la même s'appliquer à l'examen espagnol. La section suivante présente l'intégration de l'introduction en bourse a connu dans les enquêtes sur le PIB, ce qui est très 
clairement reflété dans les conclusions qui améliore l'expérience de l'introduction en bourse pour l'étude des attitudes des étudiants espagnols.

Mots-clé: Espagnol Langue Étrangère, langue étrangère, évaluation, Test General du Baccalauréat.

Structure, perception, non-linguistic factors and questions of OPI interviews in Spanish: A proposal of application to the General Test Baccalaureate

\begin{abstract}
This paper presents a study on the oral interview OPI and its application to the design of the future foreign language section in the General Test of Baccalaureate (PGB). After the presentation of the IPO and the General Test of Baccalaureate context, a study analyzing the attitudes of the candidates of that examination and extracting the basic ideas of the same to apply to the Spanish exam is presented. The following section presents the integration of the OPI experience in the research of the PGB, something that is very clearly reflected in the conclusions which enhance the experience of the OPI for the study of the attitudes of Spanish students.
\end{abstract}

Keywords: Spanish as a Foreign Language, Foreign Language, assessment, General Test of Baccalaureate.

SUMARIO 0. Introducción. 1. Propósito. 2. Fases y estructura de la opi. 3. Cuestionamientos. 4. Encuesta y análisis. 5. Aplicación de la opi a la prueba general de bachillerato. 6 . Conclusiones. Referencias bibliográficas

\title{
0. INTRODUCCIÓN
}

Este trabajo trata de acercarse a la definición y experiencia del examen OPI para su aplicación en la Prueba General de bachillerato en España. La Prueba General de Bachillerato es una evaluación de la Prueba de Acceso a la Universidad (PAU) detallada en el anteproyecto de la Ley Orgánica para la Mejora de la Calidad Educativa (LOMCE, previsiblemente 2013) y se realiza al final del Bachillerato constituyéndose en la mayor parte de los casos en un examen de acceso a la universidad. La sección de lenguas extranjeras saldrá muy beneficiada respecto al formato actual de la PAU. Por eso, es importante encontrar fundamentos e ideas en otros exámenes. Dentro de la posibilidades existentes se buscaron en la experiencia del Cambridge Board of Examinations sin embargo, como se observará, dadas sus características, la entrevista de aptitud oral OPI (Oral Proficiency Interview) resulta más adecuada para el propósito de evaluar el aprendizaje general escolar. La OPI de ACTFL se utiliza para medir globalmente la habilidad de funcionar en un idioma extranjero se ha convertido en instrumento determinante de evaluación para la mayoría de programas de idiomas en los EEUU. La OPI es realmente una evaluación de las habilidades funcionales de un hablante, mas no de su competencia lingüística, como lo establece el manual de capacitación de ACTFL, (2012): 
"...it assesses language performance in terms of the ability to use the language effectively and appropriately in real-life situations" (1). La evaluación de esta actuación y funcionalidad es global y determina patrones de fortalezas y debilidades únicas en la producción oral de cada entrevistado. Los programas universitarios que han adoptado la OPI para evaluar a sus estudiantes de último año o a quienes recién ingresan son cada vez más conscientes de sus efectos en el desarrollo de sus programas. Hoy en día las directrices de aptitud de ACTFL tienen un fuerte efecto en el contenido y la enseñanza de la metodología de muchos cursos de idiomas extranjeros. Los estándares y directrices desarrollados por ACTFL (sus "Proficiency Guidelines") son utilizados para delinear los programas de cursos de idiomas y actualmente se requiere incluirlos en los "syllabus" de cursos universitarios de lengua extranjera en los EE.UU. Dichas directrices sirven como referente para evaluar la funcionalidad de los alumnos desde el nivel principiante hasta el superior, según los descriptores para cada una de las cuatro habilidades: escuchar, hablar, leer y escribir. De acuerdo con Liskin-Gasparro, (2003) la noción de destreza comunicativa descrita por las directrices de ACTFL, ha logrado consolidarse a pesar de las críticas: "...it seems to have found its legitimacy in the arenas of policy, program development, and classroom instruction" (489).

Numerosos programas universitarios de enseñanza de lenguas requieren la entrevista OPI de ACTFL, actualmente para 37 idiomas, como instrumento de evaluación hacia la certificación de futuros profesores de idiomas. También se aplica como diagnóstico inicial o final en diversos programas, así como instrumento de nivelación o sujeto de investigación lingüística. La OPI ha sido también adoptada por instituciones no académicas para evaluar la idoneidad de candidatos a posiciones oficiales o del sector privado que pueden requerir habilidad para hablar una lengua extranjera. De acuerdo con el manual de ACTFL la OPI es un medio efectivo y confiable para medir el estándar para la comunicación interpersonal como lo describen sus delineamientos. Es también una evaluación interactiva, adaptable y centrada en el hablante, es decir: "...the active negotiation of meaning between the interviewer and interviewee to elicit a ratable simple of speech..." (3). Las aplicaciones útiles de la OPI sólo como entrevista son pocos pero lo que ella revela sobre el proceso de aprendizaje de una segunda lengua es muy valioso para desarrollar cursos que enfaticen áreas problemáticas, para que los alumnos puedan expresar su competencia oral. Lipkin-Gasparro (2007) reconocía además que “...cuando se trata del uso indirecto de las conexiones que se pueden hacer entre la escala de competencia de expresión oral y sus posibles aplicaciones pedagógicas, hay una abundancia de posibilidades tanto creativas como productivas" (28).

\section{PROPÓSITO}

El presente estudio examina el contexto e implicaciones de la OPI en español como lengua extranjera, además de considerar factores no-lingüísticos que inter- 
vienen en el desempeño del entrevistado en OPI institucionales realizadas 'cara a cara'. Inicialmente se revisa la estructura de la entrevista, fases, niveles y criterios, hasta cuestionar su fiabilidad y proponer consideraciones para reducir posibles efectos negativos y obtener una muestra más representativa. Este estudio toma en consideración la experiencia de un entrevistador de la OPI certificado por ACTFL y los juicios recogidos en una breve encuesta hecha a 10 profesores de español en preparación que tomaron la entrevista, y que aporta cuestionamientos sobre los exámenes y el proceso de aprendizaje, así como sus percepciones sobre la OPI.

\section{FASES Y ESTRUCTURA DE LA OPI}

La entrevista OPI de ACTFL consiste típicamente de cinco fases: la preliminar o "warm-up", el proceso interactivo que consta de "level checks" y "probes", para establecer el nivel y sondear a un nivel más alto, el juego de roles o "role-play", y el epílogo o "wind-down". La función del "warm-up", es hacer que el entrevistado se sienta cómodo, para familiarizarlo con la pronunciación y el acento del entrevistador, y para comenzar a generar temas que pueden ser explorados más adelante en la entrevista. El "level check" o nivel de control le permite al entrevistado demostrar su capacidad de manipular funciones y contextos en un nivel particular. Si el entrevistador está satisfecho con el rendimiento consistente del entrevistado, tratará de descubrir el "roof", o límite en un nivel más alto. Por lo tanto los "probes" o sondeos hacen que el entrevistado revele sus debilidades o inconsistencias para funcionar en el próximo nivel. La fase de "role-play", o de actuar en un contexto determinado, sirve como un escenario adicional al entrevistador para interactuar con el entrevistado y confirmar su nivel. El epílogo o "wind-down" trata de traer al entrevistado al nivel en que se sienta más cómodo y terminar la entrevista positivamente. La entrevista completa puede durar alrededor de 30 minutos, a veces más, dependiendo del nivel de aptitud del entrevistado, los sondeos de nivel y la variedad de temas discutidos. Es importante que el entrevistador sea flexible y hábil para conducir la entrevista, cambiar temas, retar el nivel del candidato y establecer un nivel base que ayude a determinar una clasificación más objetiva. El entrevistador no deberá perder el control de la entrevista, y deberá sortear, entre otras, instancias cuando el entrevistado no entiende bien e insiste en repetir animadamente un discurso aprendido sin contestar exactamente lo que se le ha preguntado. La entrevista, ya sea telefónica o institucional 'cara a cara' se graba y se manda al centro de evaluación (LTI, Testing Office de ACTFL) para cotejar la clasificación que ha concedido el entrevistador con la de un segundo calificador, y aun la de un tercero o un cuarto, si no se ha coincidido con el nivel exacto para el entrevistado.

Los niveles básicos establecidos por ACTFL para clasificar el desempeño oral de los entrevistados son cuatro: $\boldsymbol{a}$. principiante: el entrevistado puede comunicarse mínimamente mediante frases sencillas y listas aprendidas y fijas. $\boldsymbol{b}$. interme- 
dio: el entrevistado puede construir, preguntar y responder preguntas sencillas sobre temas familiares y manejar situaciones e interacciones simples. $\boldsymbol{c}$. avanzado: el entrevistado puede narrar y describir en todos los tiempos gramaticales y manejar situaciones rutinarias con facilidad. $\boldsymbol{d}$. superior: el entrevistado puede apoyar sus opiniones, hacer hipótesis, discutir concreta y abstractamente y manejar situaciones y temas complejos. En los primeros tres de ellos se han establecido tres subniveles: alto, medio y bajo. El nivel más alto o Superior ha permanecido por mucho tiempo como un nivel único, pero recientemente se ha introducido en el manual un subnivel "distinguished", o distinguido, para precisar que el entrevistado no sólo funciona eficientemente al nivel superior, sino que lo hace de manera culta manejando conocimientos y conceptos abstractos de alto orden: "Speakers at the Distinguished level produce highly sophisticated and tightly organized extended discourse. At the same time, they can speak succinctly, often using cultural and historical references to allow them to say less and mean more. At this level, oral discourse typically resembles written discours" (58).

Cabe destacar que cada uno de los cuatro niveles básicos comprende un rango de realizaciones y perfiles que representan un número de patrones de fortalezas y debilidades. También, el límite entre un nivel y otro está definido por la habilidad del entrevistado en sostener su actuación comunicativa en todas las funciones especificadas para cada nivel. Estas están determinadas por su capacidad para controlar completamente y todo el tiempo las funciones y criterios lingüísticos establecidos. El subnivel medio es el patrón más evidente para definir con claridad cada uno de los niveles básicos. Por su parte, los subniveles bajo y alto son una forma de situar la actuación del entrevistado más cerca del nivel próximo. El subnivel bajo determina que no se cumplen consistentemente las funciones del nivel básico o medio, que se notan dificultades, y que más bien la producción del entrevistado se asemeja a la superior del nivel anterior. Por su parte, el subnivel alto determina que el entrevistado no cumple consistente y sostenidamente las funciones del próximo nivel. La comisión educativa del estado de Georgia requiere que los candidatos a ser profesores obtengan al menos el nivel "Advanced Low" antes de ser certificados.

Existen cuatro criterios de evaluación en la entrevista OPI: $\boldsymbol{a}$. tareas globales o funciones realizadas en el idioma, es decir básicamente, preguntar y responder preguntas sencillas, narrar y describir y apoyar opiniones. $\boldsymbol{b}$. Contextos y áreas de contenido, o sea, circunstancias lingüísticas para realizar dichas funciones. Este criterio de evaluación normalmente hace parte de la fase de "role-play", en la que se asigna un contexto, por ejemplo, un restaurante de un país hispano y un contenido relacionado con el vocabulario de platos y bebidas. $\boldsymbol{c}$. La precisión con que se cumplen dichas funciones en los contextos establecidos, así como la comprensibilidad de cada mensaje. Este criterio se relaciona con el control que el entrevistado ejerce sobre la gramática, el vocabulario y otros elementos que hacen apropiado el mensaje 
como la precisión fonética y sintáctica. $\boldsymbol{d}$. El tipo de texto oral que produce el entrevistado al realizar las funciones comunicativas, es decir, si las realiza en segmentos, palabras o frases, párrafos narrados o en discursos extensos y articulados.

El criterio $c$. atañe más al presente estudio ya que involucra otros elementos además de la precisión lingüística del entrevistado como son su pronunciación, acento e incluso su competencia sociolingüística. Nos interesa también destacar otros factores no lingüísticos que influyen visiblemente en la manera que el entrevistado se desempeña durante una entrevista 'cara a cara' y que afectan la clasificación final del entrevistador. Factores como el nerviosismo y la ansiedad, la inseguridad por no saber suficiente, el manejo de la grabación y el micrófono, el control del tiempo, la personalidad y aun comportamientos culturales, interfieren con la producción oral del entrevistado y claro, con la percepción de su entrevistador. A este respecto ACTFL sólo advierte que los silencios no son necesariamente evidencia negativa, pero no incluye ningún otro factor. Las fases en las que más comúnmente se evidencian estos factores son durante el proceso interactivo de "level checks / probes" y el "role-play" cuando el entrevistador trata de sondear o de establecer un desempeño sostenido en la actuación del entrevistado o "floor." Sin embargo, las limitaciones lingüísticas o "ceiling" observadas en el entrevistado no deben influir la clasificación final de la OPI, de acuerdo con los descriptores para evaluadores de ACTFL. En casos excepcionales, las limitaciones lingüísticas además de confusos comportamientos no lingüísticos podrían contribuir a una clasificación de no evaluable o "unratable." En la entrevista OPI el entrevistado produce lo que él / ella percibe como más valioso para demostrar sus habilidades orales, y no obstante es, en la mayoría de los casos consciente de las fases de la OPI y consecuentemente bajo gran ansiedad o estrés. Este factor se acentúa cuando se trata de un requisito para certificación o grado, y es menor cuando sólo es una entrevista diagnóstica o parte de un examen de nivelación.

En la fase preliminar de la OPI el entrevistador normalmente explota la función descriptiva usando temas familiares como su lugar de localización u origen, su familia, sus intereses, trabajo, estudio, etc. En la OPI en español esta fase gira alrededor del uso de Ser y Estar, Haber, Gustar, Hacer, etc. El uso de estas estructuras básicas se apega más al sistema estructurado como se presenta la gramática en los textos académicos tradicionales. Luego el entrevistador procede a explotar la función narrativa del hablante solicitando información sobre actividades y experiencias cotidianas con la familia y amigos, tanto en el presente como en las formas del pasado, pretérito e imperfecto. Usualmente se le pide al entrevistado que narre con detalles de principio a fin una experiencia o anécdota memorable en el pasado, algo que le sucedió en un viaje, durante las vacaciones o en sus años escolares. Se busca aquí revelar la habilidad del entrevistado para expresarse en párrafos completos y no en frases o fragmentos aprendidos, además de exponer su control sobre formas gramaticales y de concordancia, sobre todo, género, número y 
persona. A pesar de darle una buena oportunidad al entrevistado para expresarse ampliamente, esta función puede limitarlo ya que por motivos de personalidad el entrevistado puede sentirse limitado hablando de temas o anécdotas que no recuerda en detalle o que no tiene mucho que decir. Algo similar puede ocurrir durante la segunda fase interactiva de "level checks / probes," en la que el entrevistador controla los temas y tiene ya establecidas ciertas expectativas para contra-atacar las opiniones del entrevistado. La mayoría de los temas introducidos tienen que ver con la educación, los sistemas sociales, la ecología, la economía, y aunque no se pretende evaluar el conocimiento sobre estos temas, algunos de ellos pueden limitar la expresividad del entrevistado cuando el entrevistador presiona alguna controversia. Aunque esto puede ser revelador de limitaciones lingüísticas para la OPI, el entrevistado puede percibir que estos temas no le permiten desplegar bien sus habilidades discursivas. El manual de ACTFL aconseja al entrevistador efectuar técnicas de exploración o "elicitation" como el "triple punch" que consiste en plantear una serie de preguntas en tres tandas para explotar un tema a nivel Avanzado o Superior. Las preguntas normalmente cuestionan una posición del entrevistado sobre un tema controversial, por ejemplo, el sistema escolar público, y después de retar las implicaciones del entrevistado se le pide que explique las consecuencias, costos y efectos de los cambios propuestos. Esto con el objeto de presionar su capacidad para conectar ideas fluidamente en español y defender su razonamiento o posición frente al tema. No obstante, en ocasiones algunos entrevistados pueden lamentarse de no haber podido hablar sobre temas que les gustan más, o que no estaban interesados en cuestionar temas o contradecir al entrevistador. Este caso se puede presentar cuando el entrevistado se siente presionado a dar una posición sobre algo que no le interesa, o cuando el entrevistador actúa como 'abogado del diablo' en casos como el del "triple punch." Ahora bien, cuando se trata de evidenciar el control gramatical de ciertas estructuras, es el entrevistador quien puede resultar frustrado al no obtener las respuestas esperadas con cierta estructura. Algo así se evidencia por ejemplo, al hacer una pregunta hipotética en español usando la forma pasado de subjuntivo + condicional en la misma frase, y obtener una respuesta muy general que no utiliza siquiera la forma del condicional o que en el peor de los casos no responde la pregunta. Se hace entonces necesario incluir en el manual del entrevistador ejemplos de contextos y estructuras a explotar en cada nivel y así ofrecer al entrevistador muestras que le permitan explotar más auténticamente las habilidades orales del entrevistado.

Durante la tercera fase del juego de roles o "role-play" el entrevistador introduce una situación, normalmente en tarjetas codificadas por color según el nivel y producidas por ACTFL, que presentan situaciones para cada nivel. Esta fase ofrece al entrevistador intervenir como interlocutor para escenificar situaciones que van desde simples preguntas a nivel principiante hasta temas que exigen desarrollo discursivo al nivel avanzado o superior. Las situaciones de "role-play" pueden 
involucrar implicaciones socio-lingüísticas y aun pre-concepciones culturales que pueden cohibir la libre expresión del entrevistado. En este caso así como durante las otras fases de la OPI el entrevistador debe estar presto a manejar las situaciones con tacto y cambiar si es necesario un tema o situación de "role-play". Es, por lo tanto, importante asegurarse que los temas o situaciones introducidas no contengan temas culturales o sociales sensibles que puedan afectar la disposición del entrevistado. Para llevar la OPI a feliz término el entrevistador entra a la última fase o epílogo en la que debe traer al entrevistado al terreno más cómodo de su expresión oral preguntándole principalmente sobre sus planes futuros.

\section{CUESTIONAMIENTOS}

A pesar del amplio uso de la OPI, existen cuestionamientos sobre la validez de la estructura descrita como efectiva modalidad de evaluación oral. Se cuestiona si la entrevista es una muestra fiel de la producción oral del entrevistado y si la intervención de factores no lingüísticos o circunstanciales influyen determinantemente en el entrevistado y en su clasificación. La OPI es un indicador fiable, pero de una actuación oral en circunstancias limitantes, ya que el entrevistado queda sujeto al uso de ciertas estructuras en condiciones artificiales controladas por el entrevistador. La mayoría de entrevistados encuestados, por ejemplo, coinciden en que la fase de sondeo o "level checks / probes" a través de preguntas y temas de discusión sobre los que no tienen mucho que decir los pone en situación incómoda y es un agravante de su nerviosismo. Sin embargo, existe una confusión entre muchos encuestados, (ver análisis de la encuesta más adelante), sobre la diferencia entre su competencia o conocimiento comunicativo en español y su actuación o funcionalidad real influenciada por los factores ya mencionados. Es decir, aseguran que los nervios pudieron ser los causantes principales de su cuestionable desempeño y no tanto su aptitud oral en español.

Una circunstancia especial que no ha sido muy atendida es que ACTFL no requiere que los entrevistados en una OPI sean hablantes extranjeros de la lengua en cuestión. De hecho, cada vez más candidatos a quienes se les ha requerido tomar la OPI son hablantes nativos del español o son hablantes que lo han heredado de casa como segunda lengua. En estos casos el entrevistador debe ser prudente y atenerse sólo a la producción lingüística, su clasificación no debe basarse en prejuicios o en el perfil biográfico del entrevistador, como lo advierte el manual de ACTFL para evaluadores (18). Cabe distinguir que quienes son considerados hablantes por herencia del español, por ejemplo, no tuvieron educación formal en un país hispano y su acceso a la lengua proviene principalmente de su familia inmediata en EEUU. El hecho de que un hablante suene como hablante nativo, con o sin educación formal en un país hispano hablante no garantiza una inmediata presunción de clasificarlo en el nivel Superior o Distinguised Superior. Tanto los hablantes nativos como los hablantes por herencia no conforman grupos homogé- 
neos, ya que existen diferentes niveles de cultura y educación en cada grupo. Cabe entonces considerar otro factor lingüístico relacionado con estos hablantes: su variedad dialectal en términos léxicos y fonéticos que en ocasiones podría desconcertar la percepción objetiva del entrevistador. En el protocolo de introducción a la OPI se establece que la entrevista se lleva a cabo en la lengua estándar, sin embargo, algunos entrevistados pueden no entender este concepto o pensar que se trata sólo de no hablar tan rápido. La variedad dialectal y coloquial de los entrevistados puede hacer que la evaluación de la producción oral sea en ocasiones problemática para los calificadores. Sobre la disparidad existente entre lo que los alumnos aprenden en clases formales de lengua extranjera y cómo se expresan en diversos contextos Liskin-Gasparro reconoce que no es ni su culpa ni la de de los instructores: "Es muy posible que ese tema no se aprende bien porque los alumnos simplemente no están cognitivamente listos para asimilarlo. Como se puede ver en las OPI, para algunos aspectos de la sintaxis los alumnos tardan muchos años en controlarlos bien" (29).

Ahora bien, aunque el carácter de las circunstancias involucradas en una OPI sea eminentemente artificial, tanto en la entrevista 'cara a cara' como en la telefónica, la evaluación obtenida es prácticamente la misma, como lo concluye Elvira Swender: "... an individual is likely to receive the same rating wheter tested faceto-face or telephonically, and is likely to receive the same rating when retested within a 48-hour period" (523). También, los temas de conversación introducidos en un contexto evaluativo conllevan factores de tensión y expectativa, y por la carencia de autenticidad puede ser más bien una actuación de roles, similar a la fase de "role-play." La variedad de contextos discursivos y socio-culturales de la OPI es muy limitada y puede no ser suficiente en todos los casos para evaluar la funcionalidad comunicativa de un entrevistado. En el caso de la entrevista 'cara a cara' o institucional el entrevistado puede ya conocer a su entrevistador, pero en el caso de la entrevista telefónica puede resultar aún más artificial compartir opiniones con un desconocido, a quien no puede ver ni se sabe cómo dirigirse, si de Tú o Usted. (Para una revisión en español de las críticas y cuestionamientos a la OPI desde su introducción véase el estudio de Alfonso Martínez, "La evaluación oral: una equivalencia entre las guidelines de ACTFL y algunas escalas del MCER" http://www.atriumlinguarum.org/contenido/EscalasEvaluaci\%C3\%B3n.pdf).

\section{ENCUESTA Y ANÁLISIS}

Las respuestas obtenidas en la encuesta (ver encuesta en el Apéndice) a 10 alumnos hablantes de inglés, con especialidad en educación de español, en Valdosta State University que ya han tomado la OPI dan una clara muestra de sus percepciones sobre los exámenes y el aprendizaje, y sobre la OPI. Sobre la primera parte de la encuesta, la evaluación general por exámenes el $80 \%$ de los alumnos encuestados demuestra no estar seguros sobre las diferencias metodológicas de enfoques 
tradicionales como el eminentemente basado en la gramática, el comunicativo y uno ecléctico y marcaron alguno o los tres al tiempo (item 1). La mayoría de los encuestados, tampoco está segura sobre el impacto de los exámenes en el proceso de aprendizaje de una lengua extranjera y sus opiniones fueron divididas $60 \%$ / $40 \%$ (item 2). A pesar de haber tomado ya al menos tres exámenes estándares tipo test, el $50 \%$ de los encuestados afirman no haber estado más ansiosos por pasar los exámenes que por aprender metodología de la enseñanza (items 3, 4). Podría esto deberse a su concepción sobre la cultura educativa en la que no se puede prescindir de exámenes estándar por 'tests', aun para medir la aptitud comunicativa en una lengua extranjera. El $60 \%$ de los encuestados afirmaron estar familiarizados con algunos formatos generales de los exámenes estándar, y agregaron la ansiedad adicional por no tener que repetir los que cuestan bastante dinero (item 5). Finalmente, a propósito de su habilidad para aplicar eficientemente técnicas de evaluación a sus futuros alumnos el $90 \%$ de los encuestados afirmaron positivamente (item 6).

Ahora bien, sobre aspectos específicos de la entrevista OPI, en la segunda parte de la encuesta, el $90 \%$ de los alumnos respondieron positivamente sobre su familiaridad con los estándares y directrices de ACTFL antes de tomar la OPI (ítem 1). El 50\% de los encuestados admitieron no haber tomado ningún curso preparatorio para el OPI, a pesar de que algunos sí habían tomado un curso de conversación intermedia en su segundo año de español en VSU, (ítem 2). Con respecto al tema de los factores no lingüísticos (ítem 3) el 100\% de ellos coincidió en ubicar el nerviosismo como el factor principal a la hora de tomar la entrevista, seguida por su inseguridad de no saber suficiente español. Otra vez la gran mayoría, el 90\%, estuvo de acuerdo con: sentirse cómodo con la secuencia y fases de la entrevista, (ítem 4). Sin embargo, creen haberse sentido menos a gusto durante la fase de sondeo para establecer el nivel o "level checks/probes," sólo la fase de juego de roles o "role-play" fue incluida una vez, (ítem 5). El 100\% de los encuestados creen que la entrevista OPI evalúa de forma confiable su potencial de expresión oral, (ítem 6), aunque por lo general se sienten mejor con la OPI 'cara a cara' que con una eventual entrevista telefónica, (ítem 7). El 90\% de ellos también coincide con que la OPI debe ser un requisito para que los profesores de lengua extranjera se gradúen o logren su certificación, (ítem 8). Finalmente, con respecto a usar su experiencia con la OPI en la evaluación de sus futuras clases el 100\% de los encuestados respondieron afirmativamente, (ítem 9), aunque no todos estuvieron seguros de incluir o sugerir otros elementos que se podrían incluir en evaluaciones orales tales como la OPI, (ítem 10). La única sugerencia tuvo que ver con incluir, hacia una evaluación más confiable, una OPI en la L1 del entrevistado, para que los evaluadores puedan eficientemente distinguir las limitaciones lingüísticas de los rasgos culturales o de personalidad, o factores negativos no lingüísticos. Algunos entrevistados pueden hacer pausas más largas que no sólo pueden reflejar inseguridad 
sino una reflexión más amplia o cuidadosa de sus ideas o expresión, factores que no se dan de igual manera en todos ellos. Sin embargo, la disponibilidad de profesores perfectamente bilingües para conducir entrevistas en dos idiomas, el tiempo y los costos adicionales incurridos hacen que esta opción no se haga factible.

\section{APLICACIÓN DE LA OPI A LA PRUEBA GENERAL DE BACHILLE- RATO}

Entre los numerosos cambios que el Ministerio de Educación, Cultura y Deporte pretende llevar a cabo en el sistema educativo español se encuentra la propuesta de introducir una prueba oral de inglés en la antigua Prueba de Acceso a la Universidad - que, como se dijo en la introducción, pasará a llamarse Prueba General de Bachillerato (PGB)- con el objetivo de que los alumnos españoles mejoren su competencia oral en el idioma extranjero.

El Real Decreto que regulaba las condiciones de acceso a las enseñanzas universitarias oficiales de grado y los procedimientos de admisión a las universidades públicas españolas, aprobado en 2008, establecía un examen oral de lengua extranjera para este curso 2011-2012, pero finalmente el Consejo Escolar del Estado decidió aplazarlo por las dificultades para su aplicación además de la "necesidad de elaborar un protocolo de actuación que permita garantizar la objetividad en la evaluación y los derechos que asisten al alumnado en referencia a dicha prueba".

La propuesta de introducción de una entrevista similar a la OPI (Oral Proficiency Interview) al finalizar la etapa de bachillerato toma sentido al observar de cerca el Estudio Europeo de Competencia Lingüística en la Unión Europea (EECL), publicado en julio de 2012, que recalca la mala posición de los españoles frente a la mayoría de los países europeos en el dominio del inglés -sobre todo en comprensión oral- y hace hincapié en la importancia de las lenguas extranjeras en el sistema educativo para facilitar la transición de los estudiantes al mundo universitario y laboral.

El Ministerio de Educación decidió experimentar, a pesar del retraso en su implantación, en algunas comunidades que habían iniciado ya el proceso y que han manteniendo la prueba en proceso experimental, como es el caso de Aragón, Islas Baleares, La rioja, Madrid, Castilla-La Mancha, Asturias y Comunidad Valenciana. Paralela a la investigación del ministerio, además, proyectos como OPENPAU (MINECO-FEDER Humanidades, FFI2011-22442,2012-2014), defienden la implantación de la prueba y trabajan para tratar de conseguir su efectividad y validez.

\section{CONCLUSIONES}

La OPI logra evaluar parcialmente las habilidades comunicativas del entrevistado al exponer buena parte de su fluidez discutiendo ideas, su precisión gramatical y su control sintáctico. Las limitaciones inherentes a la entrevista parten de las circunstancias evaluativas en las que se encuentra el entrevistado y de su relación 
con quien ostenta el control total de la conversación en un contexto artificial. El entrevistado depende completamente del entrevistador y de la estructura basada en las fases de la entrevista, él es quien introduce los temas y ya tiene no sólo un plan estructurado sino expectativas sobre el rango de respuestas obtenidas que le permitan explotarlas, a veces exhaustivamente, aunque no sea nada interesante para el entrevistador ahondar demasiado. Entonces, es cuestionable que la evaluación del candidato dependa en gran forma de una estructura pre-concebida con el propósito de revelar limitaciones lingüísticas, que pueden también limitar su rango de expresividad. Como consecuencia del carácter evaluativo de la entrevista los candidatos que no alcanzan el nivel requerido pueden desanimarse y desinteresarse por continuar estudiando español. Por tanto, lo que el entrevistado percibe como una mala experiencia al tomar su OPI no necesariamente revela su ineptitud para hablar el idioma sino las características limitantes, inherentes de la entrevista, y las circunstancias no lingüísticas presentes ese día.

Siendo la OPI, como lo será una parte de la PGB, una evaluación de destrezas orales dentro de los parámetros limitantes de la entrevista es necesario considerarla como tal y no como una prueba definitiva de competencia orales para conversar en L2. Así las cosas, al no representar la OPI en todos los casos una muestra integral de la capacidad comunicativa de un hablante en una lengua extranjera debe considerársele sólo como un componente evaluativo de las funciones orales que propone. Resulta entonces optimista pensar que ACTFL diseñe una entrevista que evalúe integralmente competencia y actuación lingüística, así como sus componentes gramaticales y discursivos, en circunstancias sociolingüísticas, culturales y de personalidad ideales. A pesar de las críticas la OPI se ha ido mejorando y sigue vigente como la mejor alternativa de evaluación oral, a pesar de las implicaciones de factores no lingüísticos.

Algunas de nuestras recomendaciones tienen que ver con la aplicación de las directrices de ACTFL en cursos universitarios de conversación intermedia con énfasis en la familiarización y práctica de la estructura de la OPI. Así pues, la aplicación de la OPI a la PGB busca desarrollar en los alumnos las destrezas orales que les permita desenvolverse con facilidad en las diferentes fases de la entrevista, explorando las posibilidades lingüísticas de cada función. Esto se aplica especialmente para estudiantes que tendrán que tomar la PGB oficial en su último curso o, como en la aplicación en EEUU, requisito de certificación para futuros profesores de secundaria. Este curso deberá constar también con entrevistas simuladas como método de práctica una a mitad y otra al final del semestre. De este modo se reduce el factor inseguridad y ansiedad que generan el nerviosismo de quienes no saben qué esperar de la entrevista. Ahora bien, en casos que se requiera una evaluación oral más integral se agregaría a la entrevista una introducción, en inglés, de unos cinco minutos para que el entrevistador se haga una imagen de la personalidad y capacidad discursiva del entrevistado al darle un tema de opinión para discu- 
tir. De esta manera el entrevistado logra asumir una actitud más relajada y estar más dispuesto a expresarse en inglés o en español (en la OPI). Esta variación ha resultado conveniente en entrevistas simuladas, 'cara a cara' y les ha probado a los investigadores ser un recurso efectivo para lograr una mejor disposición comunicativa y una muestra más auténtica del entrevistado. En el caso de las entrevistas OPI o, incluso la PGB, por teléfono, una variedad recomendable sería hacerla usando el recurso del video que proveen programas de comunicación en línea, para así personalizar la interacción y reducir la ansiedad inherente que produce ser evaluado.

Nuestro agradecimiento al Ministerio de Economía y Competitividad por la financiación del proyecto de investigación (con cofinanciación FEDER) en el marco del Plan Nacional I+D $+\mathrm{I}$ «Orientación, propuestas y enseñanza para la sección de inglés en la Prueba de Acceso a la Universidad». Referencia FFI2011-22442.

\section{REFERENCIAS BIBLIOGRÁFICAS}

American Council on the Teaching of Foreign Languages. Swender E. \& R. Vicars, Eds. Oral Proficiency Interview Tester Training Manual. White Plains, NY (2012).

LISKIN-GASPARRO, Judith. "La evaluación de destrezas orales: Perspectivas norteamericanas". Actas del XVII Congreso Internacional de ASELE, Universidad de La Rioja, Logroño 2007. (19-38)

LISKIN-GASPARRO, Judith. "The ACTFL Proficiency Guidelines and the Oral Proficiency Interview: A Brief History and Analysis of Their Survival." Foreign Language Annals. Vol 36, No. 4. 2003. (483-490).

SwENDER, Elvira. "Oral Proficiency Testing in the Real World: Answers to Frequently Asked Questions". Foreign Language Annals. Vol. 36, No. 4. 2003. (520-526) 


\section{ENCUESTA}

\section{General Perceptions by Spanish FLED majors towards testing and the OPI}

Semester you took the OPI:

\section{General questions}

1. Thus far, to which language teaching style have you been exposed?
a. grammar-translation based
b. communicative-functional
c. eclectic

2. In your opinion, can testing negatively impact actual learning of a foreign /second language?
a. yes
b. no
explain:

3. During your preparation to become a teacher, what required tests have you taken?
a. oral:
b. written:
c. none.

4. Have you been more anxious about passing tests than learning about practical teaching methods?
a. yes
b. no
explain:

5. Are you already familiar with testing formats to assess all language skills?
a. yes
b. no
explain:

6. Do you think that you will be prepared enough to efficiently assess your future language students?
a. yes
b. no
explain: 


\section{On the Institutional OPI}

1. Were you familiar with the ACTFL standards and Proficiency guidelines before taking the OPI?
a. yes
b. no
explain:

2. Did you take a specific course preparing you for the OPI?
a. yes
b. no
explain:

3. What non-linguistic factors might have influenced your performance during your OPI?
a. nerves
b. insecurity
c. time constraint anxiety
d. other:

4. Did you feel comfortable with the sequence and different phases of the interview?
a. yes
b. no
explain:

5. During which phase did you feel least comfortable expressing your oral potential?
a. warm-up
b. level checks/probes
c. role-play
d. wind-down

6. Do you think that the OPI reliably assesses your oral potential?
a. yes
b. no
explain:

7. Could you have performed better if you had taken the OPI as a phone interview?
a. yes
b. no
explain:

8. Should the OPI be a required test to graduate or to get your teaching certification?
a. yes
b. no
explain:

9. Do you think you could use your experience with the OPI in future class assessments?
a. Yes
b. no explain:

10. In your opinion, what other elements, if any, should be included in oral assessments such as the OPI? 